\title{
Hysteretic Nonlinearity Observer Design Based on Kalman Filter for Piezo-actuated Flexible Beams with Control Applications
}

\author{
Teerawat Sangpet ${ }^{1} \quad$ Suwat Kuntanapreeda ${ }^{1} \quad$ Rüdiger Schmidt ${ }^{2}$ \\ ${ }^{1}$ Department of Mechanical and Aerospace Engineering, King Mongkut's University of Technology North Bangkok, Bangkok 10800, Thailand \\ ${ }^{2}$ Institute of General Mechanics, RWTH Aachen University, Aachen D-52056, Germany
}

\begin{abstract}
Piezoelectric actuators fundamentally possess hysteresis behavior. Estimation of the hysteresis is usually demanded for enhancing the performance of piezo-actuated systems. This paper presents an observer-based scheme to estimate the hysteresis in piezo - actuated flexible beams. The observer is based on a nonlinearity observer method. The discrete-time Kalman-filter algorithm is adopted for determination of the observer gains. The major advantages of the presented scheme include ease of implementation and robustness to uncertainty of hysteresis parameters. Simulation results demonstrate that the observer is able to estimate the hysteresis efficiently and has better robustness compared to the previous scheme existing in the literature. The present scheme was also successfully applied to a real-life system. Moreover, a control application example is included to demonstrate the effectiveness of the scheme.
\end{abstract}

Keywords: Observers, piezoelectric actuator, Kalman filter, hysteresis, hysteresis compensation, flexible beam.

\section{Introduction}

Piezoelectric actuators have drawn extensive attention in modern industrial applications. Their advantages include nanometer scale resolution, high stiffness, and fast response. Examples of the applications include micro/nano positioning systems ${ }^{[1,2]}$ and smart structures ${ }^{[3,4]}$. However, the piezoelectric actuators fundamentally exhibit hysteresis behavior in response to their voltage excitation. This behavior usually leads to deterioration in positioning accuracy and operation performance. The hysteretic effect can be attenuated using nonlinearity compensation techniques. The techniques usually make use of hysteresis models. Some examples of the hysteresis models include the Preisach model ${ }^{[5,6]}$, the Prandtl-Ishlinskii model ${ }^{[7,8]}$, and the Bouc-Wen model ${ }^{[9,10]}$.

Observers for nonlinear systems have attracted considerable attentions for many decades due to their various high potential applications. Some examples of the applications include fault diagnosis ${ }^{[1]}$, unknown input estimation ${ }^{[12]}$, state estimation $^{[13]}$, and nonlinearity compensation ${ }^{[14,15]}$.

Nonlinearity compensation techniques have been successfully adopted for minimizing the effect of nonlinearities (such as friction, hysteresis, disturbance, etc.) in control systems. In principle, the nonlinearities first are estimated and the results are then used in the control law to compensate the nonlinearities. Freidovich et al. ${ }^{[14]}$ studied friction compensation in tracking control of a direct current (DC) motor. The friction is estimated by an observer, which is designed based on a dynamic LuGre model. The experiments demonstrate the effectiveness of the model-based ob-

\footnotetext{
Regular paper

Manuscript received February 15, 2013; accepted November 15, 2013

This work was supported by Royal Golden Jubilee Ph. D. Program of the Thai Research Fund.
}

server compensation. Liu et al. ${ }^{[15]}$ investigated a stabilization problem for a class of nonlinear systems with disturbance. An observer is used to estimate disturbance approximately. The bound of the observer error is unknown, and an adaptive sliding mode controller is designed to make the state of the control system converge to zero asymptotically. Wiener ${ }^{[16]}$ developed a method to compensate hysteresis in pneumatic actuators of optical elements in microlithography tools. The hysteresis estimator is based on the Krasnoselskii-Pokrovskii operators. The experimental results show that the method efficiently improves the precision positioning as desired.

As far as hysteresis compensation for piezoelectric actuated systems is concerned, $\mathrm{Ru}$ et al. ${ }^{[17]}$ presented an adaptive inverse control approach based on the Prandtl-Ishlinskii hysteresis model for piezoelectric actuators. The hysteresis model is derived by the identification of least mean square adaptive algorithm. The experimental results show that the position precision is noticeably improved in open-loop operation compared to the conventional open-loop control without any compensation. Lin and Yang ${ }^{[18]}$ proposed a hysteresis observer based on a Bouc-Wen model for precise positioning of a piezoelectric actuator. The observer estimating the hysteresis effect is used for hysteresis compensation. The observer error is solved by means of a proportional integral (PI) feedback control strategy to enhance the robustness of the control system. Juhász et al. ${ }^{[19]}$ developed a hysteresis compensation method based on the Maxwell resistive capacitor model. The method is successfully applied to the piezoelectric actuators incorporated in a hybrid micropositioning system. Liu et al. ${ }^{[20]}$ presented a feedforward approach based on the inverted Preisach model for compensation of the hysteresis in piezoelectric actuators. The singular value decomposition-based least squares estimation is used for the identification of the parameters of 
the Preisach model.

This paper presents work that makes use of a nonlinearity observer developed by Müller ${ }^{[21-23]}$ to estimate the hysteresis in a piezo-actuated flexible beam. The observer does not require any hysteresis models. Thus, it is more robust compared to model-based observer schemes. The discrete-time Kalman filter is adopted to obtain the gains of the observer to avoid empirical tuning of the gains. Both simulation and experimental studies, including control applications, were conducted in the paper. Note that the Kalman filter was previously employed for vibration control of piezo-actuated flexible beams in [24]. However, unlike in our work, the filter is designed to estimate the state variables of the control plant and the hysteresis was not included in the plant.

The rest of the paper is organized as follows. Section 2 reviews some preliminaries including the nonlinearity observer and the Bouc-Wen hysteresis model. The proposed Kalman-filter based nonlinearity observer is given in Section 3. Section 4 describes the piezo-actuated flexible beam that is used as an experimental test bench. Simulation studies are presented in Section 5. The results are compared with those obtained by the previous observer existing in the literature. Section 6 contains presentation of experiments. Application examples for a control problem are also given in Sections 5 and 6 . The last section concludes the paper.

\section{Preliminaries}

\subsection{Nonlinearity observer}

The nonlinearity observer developed in $[21-23]$ is reviewed in this subsection. Consider the following nonlinear system

$$
\begin{aligned}
& \dot{x}(t)=A x(t)+N f(x(t), t)+B u(t) \\
& y(t)=C x(t)
\end{aligned}
$$

where $x, u$, and $y$ are the state vector, control vector, and the output vector, respectively. $A, B, C$ and $N$ are the system matrix, control input matrix, output matrix, and nonlinearity matrix, respectively. $f(\cdot)$ is an unknown nonlinear function. The nonlinearity observer is designed to estimate $f(\cdot)$.

The fundamental idea of the observer is to approximate $f(\cdot)$ by a fictitious system

$$
\begin{aligned}
& f(x(t), t) \approx H v(t) \\
& \dot{v}(t)=V v(t) .
\end{aligned}
$$

By substituting (2) into (1), the observer is chosen as

$$
\begin{aligned}
{\left[\begin{array}{c}
\dot{\hat{x}} \\
\dot{\hat{v}}
\end{array}\right]=} & {\left[\begin{array}{cc}
A & N H \\
0 & V
\end{array}\right]\left[\begin{array}{c}
\hat{x} \\
\dot{\hat{v}}
\end{array}\right]+\left[\begin{array}{l}
B \\
0
\end{array}\right] u+} \\
& {\left[\begin{array}{c}
L_{x} \\
L_{v}
\end{array}\right](y-C \hat{x}) }
\end{aligned}
$$

where $L_{x}$ and $L_{v}$ are the observer gain matrices that must be chosen such that the observer is asymptotically stable.

In a special case, when $H=I$ and $V=0$ are chosen, the observer is reduced to a proportional-integral (PI) observer

$$
\begin{aligned}
& \dot{\hat{x}}=A \hat{x}+B u+L_{x}(y-C \hat{x})+L_{v} \int(y-C \hat{x}) \mathrm{d} t \\
& \hat{v}=-L_{v} \int(y-C \hat{x}) \mathrm{d} t
\end{aligned}
$$

and the estimated nonlinearity is given by

$$
\hat{f}(t)=\hat{v}(t) .
$$

The PI observer is successfully applied to control problems $^{[25,26]}$. The reader is referred to [21-23] for the proof of the estimation convergence and the analysis of the estimation errors.

\section{$2.2 \quad$ Assumed-modes model}

In this subsection, an approximated state-space model of piezo-actuated flexible cantilever beams based on the assumed modes method is given. The reader is referred to Gosavi and Kelkar ${ }^{[27]}$ for a full derivation of the model.

Consider a piezo-actuated flexible cantilever beam with the uniform cross section $A$ and the length $L$. The piezoelectric actuator of length $l_{p}$ is bonded at the location $x_{p}$. The tip displacement of the beam and the voltage applied to the piezoelectric actuator are denoted as $y(t)$ and $u(t)$, respectively. When the hysteresis nonlinearity of the piezoelectric actuator is ignored, the approximated state-space model using only the first $N$ modes of vibration is given as

$$
\begin{aligned}
& \dot{x}(t)=\mathrm{A} x(t)+B u(t) \\
& y(t)=C x(t)+D u(t)
\end{aligned}
$$

where $x(t)=\left[\begin{array}{lllllll}q_{1} & \dot{q}_{1} & q_{2} & \dot{q}_{2} & \cdots & q_{N} & \dot{q}_{N}\end{array}\right]^{\mathrm{T}}$ is the state vector of the generalized coordinates $q_{i}$ and $A, B, C$, and $D$ are the matrices given as follows:

$$
\begin{aligned}
& A=\left[\begin{array}{ccccc}
0 & 1 & \cdots & 0 & 0 \\
-\omega_{1}^{2} & -2 \varsigma_{1} \omega_{1} & \cdots & 0 & 0 \\
\vdots & \vdots & \ddots & \vdots & \vdots \\
0 & 0 & \cdots & 0 & 1 \\
0 & 0 & \cdots & -\omega_{N}^{2} & -2 \varsigma_{N} \omega_{N}
\end{array}\right] \\
& B=\frac{C_{a}}{\rho A L^{3}}\left[\begin{array}{c}
0 \\
\phi_{1}^{\prime}\left(x_{p}\right)-\phi_{1}^{\prime}\left(x_{p}+l_{p}\right) \\
\vdots \\
0 \\
\phi_{N}^{\prime}\left(x_{p}\right)-\phi_{N}^{\prime}\left(x_{p}+l_{p}\right)
\end{array}\right] \\
& C=\left[\begin{array}{lllll}
\phi_{1}(L) & 0 & \cdots & \phi_{N}(L) & 0
\end{array}\right] \\
& D=[0] \text {. }
\end{aligned}
$$

Here $\omega_{i}, \zeta_{i}$, and $\phi_{i}(x)$, respectively, are the natural frequency, damping ratio, and mode shape of the $i$-th mode of vibration. $\rho$ is the density of the beam material. $C_{a}$ is a geometric constant of the piezoelectric actuator.

\subsection{Bouc-Wen hysteresis model}

By considering only the first mode of the beam, the model (6) with the hysteretic effect based on the Bouc-Wen hys- 
teresis model ${ }^{[9,10]}$ can be expressed as

$$
\begin{aligned}
& \ddot{y}+2 \zeta \omega_{n} \dot{y}+\omega_{n}^{2} y=\omega_{n}^{2}(d u-h) \\
& \dot{h}=\alpha d \dot{u}-\beta|\dot{u}| h-\gamma \dot{u}|h|
\end{aligned}
$$

where $h$ is the hysteresis variable, $\alpha, \beta, \gamma$ are the parameters of the hysteresis loop, and $d$ is the displacement-voltage ratio.

\section{Kalman-filter based nonlinearity ob- server}

To implement the nonlinearity observer described in the previous section, the observer gain matrices $L_{x}$ and $L_{v}$ must be chosen. Here, instead of using fixed $L_{x}$ and $L_{v}$, the discrete-time Kalman filter algorithm ${ }^{[28,29]}$ is adopted to determine the gain matrices. By doing this, the benefit of the Kalman filter (as an optimal estimator) can be easily taken. The algorithm can be summarized as follows.

First, express (3) in a form of discrete-time representations, for example, using a zero-order hold discretization method $^{[30]}$ as

$$
\begin{aligned}
{\left[\begin{array}{c}
\hat{x}(k+1) \\
\hat{v}(k+1)
\end{array}\right]=} & {\left[\begin{array}{cc}
A_{d} & N_{d} H_{d} \\
0 & V_{d}
\end{array}\right]\left[\begin{array}{c}
\hat{x}(k) \\
\hat{\hat{v}}(k)
\end{array}\right]+\left[\begin{array}{c}
B_{d} \\
0
\end{array}\right] u(k)+} \\
& {\left[\begin{array}{c}
L_{x, d} \\
L_{v, d}
\end{array}\right]\left(y(k)-C_{d} \hat{x}(k)\right) }
\end{aligned}
$$

where $k$ is the time-step index and the subscript $d$ denotes discrete-time equivalent matrices. Then, the discrete-time state prediction is given by

$$
\bar{X}(k+1)=\Phi \hat{X}(k)+\Delta u(k)
$$

where $\bar{X}(k)=\left[\begin{array}{c}\bar{x}(k) \\ \bar{v}(k)\end{array}\right]$ is the prediction at the time-step $k$, $\hat{X}(k)=\left[\begin{array}{l}\hat{x}(k) \\ \hat{v}(k)\end{array}\right]$ is the estimation at the time-step $k, \Phi=$ $\left[\begin{array}{cc}A_{d} & N_{d} H_{d} \\ 0 & V_{d}\end{array}\right]$, and $\Delta=\left[\begin{array}{c}B_{d} \\ 0\end{array}\right]$. The state correction is given by

$$
\hat{X}(k)=\bar{X}(k)+K(k)(y(k)-\Psi \bar{X}(k))
$$

where $\Psi=\left[C_{d} 0\right]$ and $K(k)=\left[\begin{array}{c}L_{x, d}(k) \\ L_{v, d}(k)\end{array}\right]$ is known as the Kalman gain. The gain $K(k)$ is determined as

$$
K(k)=\bar{P}(k) \Psi^{\mathrm{T}}\left(\Psi \bar{P}(k) \Psi^{\mathrm{T}}+R\right)^{-1}
$$

where $R$ denotes the measurement noise covariance and $\bar{P}(k)$ satisfies the recursion

$$
\begin{aligned}
& P(k)=(I-K(k) \Psi) \bar{P}(k) \\
& \bar{P}(k+1)=\Phi P(k) \Phi^{\mathrm{T}}+Q .
\end{aligned}
$$

Here $Q$ denotes the process noise covariance and $I$ is the identity matrix with appropriate dimension.

\section{Experimental system}

\subsection{System description}

This subsection describes the piezo-actuated beam that is used as an experimental test bench. A schematic and a photograph of the experimental system is shown in Fig. 1. The system consists of a flexible cantilever beam, a piezoelectric actuator, a high-voltage power amplifier, a laser displacement sensor, a 12-bit A/D and D/A interface board and a PC. The beam is made of aluminum. The dimensions and properties of the beam are summarized in Table 1 . The piezoelectric actuator is lead-zirconate-titanate (also known as PZT). It is bonded on the beam at the distance $10 \mathrm{~mm}$ from the clamped point. The dimensions and properties of the actuator are summarized in Table 2. The tip displacement of the beam is measured by the laser displacement sensor. The displacement signal is fed to the computer through the interface board. The input voltage signal from the computer is converted to an analogue signal by the interface board, and is transmitted to the amplifier. The gain of the amplifier is 150 .

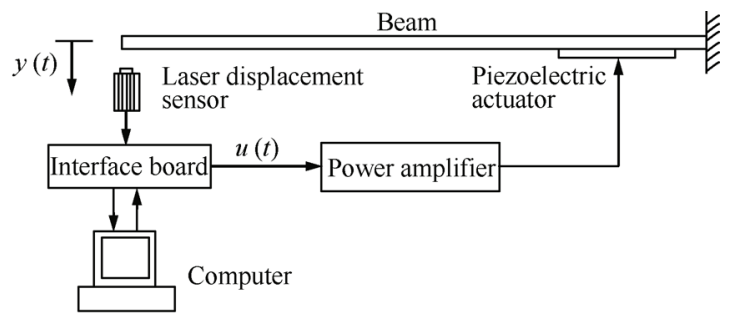

(a) Schematic

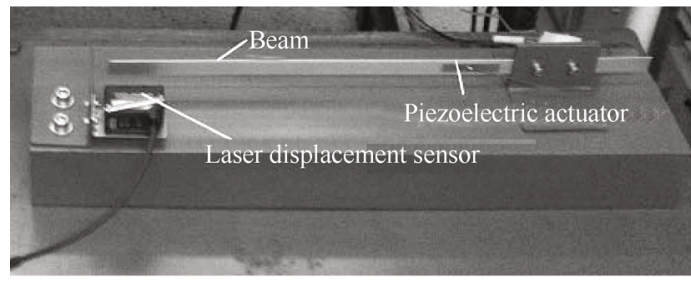

(b) Photograph

Fig. 1 Experimental system

Table 1 Beam's dimension and properties

\begin{tabular}{cc}
\hline Dimensions/Properties & Value \\
\hline Young's modulus $\left(E_{b}\right)$ & $59 \mathrm{GPa}$ \\
Density $\left(\rho_{b}\right)$ & $2700 \mathrm{~kg} / \mathrm{m}^{3}$ \\
Poisson ratio $(\nu)$ & 0.35 \\
Length $(L)$ & $0.335 \mathrm{~m}$ \\
Width $\left(b_{b}\right)$ & $15 \mathrm{~mm}$ \\
Thickness $\left(t_{b}\right)$ & $0.8 \mathrm{~mm}$ \\
Viscous damping $(B)$ & $0.1 \mathrm{~kg} / \mathrm{ms}$ \\
Structural damping $(C)$ & $0.04 \mathrm{~kg} / \mathrm{s}$ \\
\hline
\end{tabular}


Table 2 Piezo-material's dimensions and properties

\begin{tabular}{cc}
\hline Dimensions/Properties & Value \\
\hline Young modulus $\left(E_{p}\right)$ & $52 \mathrm{GPa}$ \\
Density $\left(\rho_{p}\right)$ & $7800 \mathrm{~kg} / \mathrm{m}^{3}$ \\
Length $\left(l_{p}\right)$ & $48 \mathrm{~mm}$ \\
Width $\left(b_{p}\right)$ & $10 \mathrm{~mm}$ \\
Thickness $\left(t_{p}\right)$ & $0.5 \mathrm{~mm}$ \\
Strain Coefficient $\left(d_{33}\right)$ & $390 \mathrm{e}^{-12} \mathrm{~m} / \mathrm{V}$ \\
Strain Coefficient $\left(d_{31}\right)$ & $-190 \mathrm{e}^{-12} \mathrm{~m} / \mathrm{V}$ \\
Voltage Coefficient $\left(g_{33}\right)$ & $24.0 \mathrm{e}^{-3} \mathrm{~V} \cdot \mathrm{m} / \mathrm{N}$ \\
Voltage Coefficient $\left(g_{31}\right)$ & $-11.6 \mathrm{e}^{-3} \mathrm{~V} \cdot \mathrm{m} / \mathrm{N}$ \\
Coupling Coefficient $\left(k_{33}\right)$ & 0.72 \\
Coupling Coefficient $\left(k_{31}\right)$ & 0.35 \\
\hline
\end{tabular}

\subsection{Parameter determination}

The model (7) is adopted as a representation of the experimental system. The parameters $\omega_{n}$ and $\zeta$ were directly determined by considering the free vibration responses of the beam. The parameters $d, \alpha, \beta, \gamma$ were determined by tuning in simulations (see Section. 5 for simulation details) and comparing to the actual hysteresis loop obtained from the experimental system (see Fig. 2). The input was set as $u(t)=\sin (10 t)$ volts. The determined parameters are summarized in Table 3.

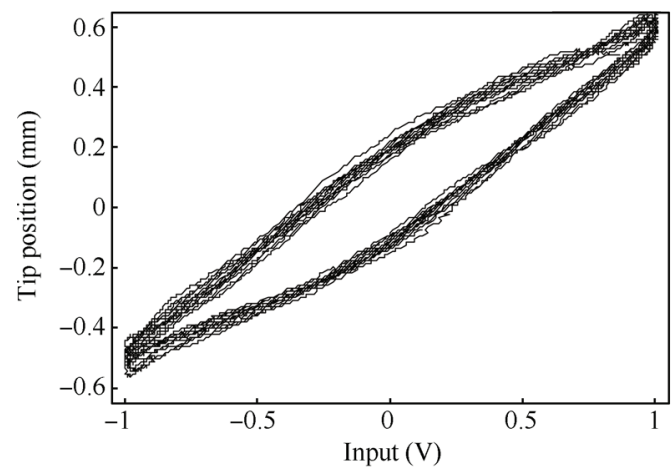

(a) Experimental result

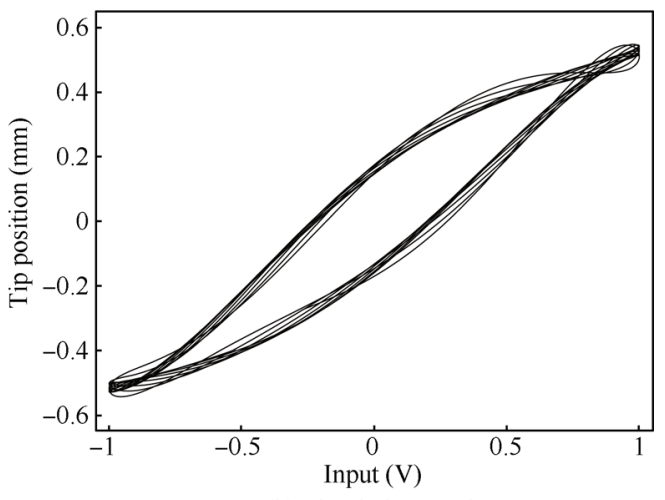

(b) Simulation result

Fig. 2 Hysteresis loops
Table 3 Determined parameters

\begin{tabular}{cc}
\hline Parameters & Values \\
\hline Natural frequency $\left(\omega_{n}\right)$ & $39.0311 \mathrm{rad} / \mathrm{s}$ \\
Damping ratio $(\zeta)$ & 0.0097 \\
Displacement-voltage ratio $(d)$ & 0.64 \\
Loop parameters $(\alpha, \beta, \gamma)$ & $0.5,1.5,0.9$ \\
\hline
\end{tabular}

\section{Simulation studies}

The model (7) with the parameters in Table 3 is used as a controlled system in the simulation studies. The studies were conducted in Matlab using 4th-order Runge-Kutta method with the fixed step size of $0.0001 \mathrm{~s}$. The sampling period for implementing the Kalman filter is $0.001 \mathrm{~s}$. The process noise covariance and the measurement noise covariance are chosen as $Q=\left[\begin{array}{ccc}1 & 0 & 0 \\ 0 & 1 & 0 \\ 0 & 0 & 1 \times 10^{5}\end{array}\right]$ and $R=1$, respectively. The observer parameters are set as $H_{d}=I$ and $V_{d}=I$, equivalent to $H=I$ and $V=0$ for the continuous-time representation. The estimated state vector is $\hat{X}^{\mathrm{T}}=\left[\begin{array}{lll}\hat{y} & \dot{\hat{y}} & \hat{h}\end{array}\right]$. The input is set as $u(t)=\sin (10 t)$ volts. The hysteresis loop estimated by the observer is shown in Fig. 3, which is almost identical to the hysteresis loop in Fig. 2(b). This demonstrates that the present observer estimates the hysteresis loop effectively. Fig. 4 displays the estimated hysteresis variable $\hat{h}$ versus the true one. In the figure, the result is also compared to the one obtained by using the Bouc-Wen model based observer developed in [18], assuming the hysteresis parameters are exactly known. The results show that both observers effectively estimate the hysteresis variables. In the top figure, it can be seen that the present observer converges faster, but with larger oscillation behavior. The convergences of the Kalman gains $K^{\mathrm{T}}=\left[\begin{array}{lll}L_{1} & L_{2} & L_{3}\end{array}\right]$ are shown in Fig. 5 . The gains converge within $0.01 \mathrm{~s}$.

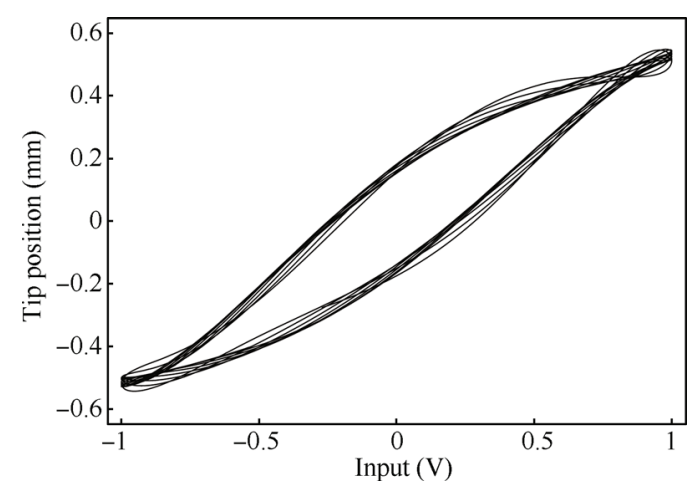

Fig. 3 Simulation result of estimated hysteresis loop

To test the robustness of the observers concerning the uncertainties of the hysteresis parameters, the parameter $\alpha$ of the system was increased by $50 \%$ (i.e., from 0.5 to 0.75 ) while $\alpha$ used in the Bouc-Wen model observer remained the same. The results are shown in Fig. 6. It shows that the present observer still estimates the hysteresis effectively, but there is large error when using the Bouc-Wen based observer. Similar results are also found when other parameters 
were changed (see Figs. 7-9). This is a major advantage of the proposed non-model-based observer.
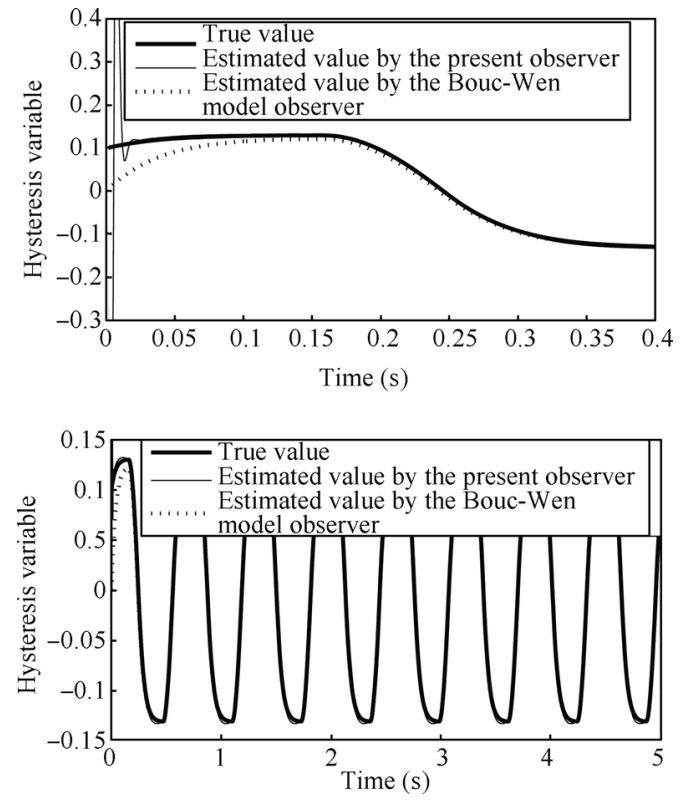

Fig. 4 Simulation result of estimated hysteresis variable
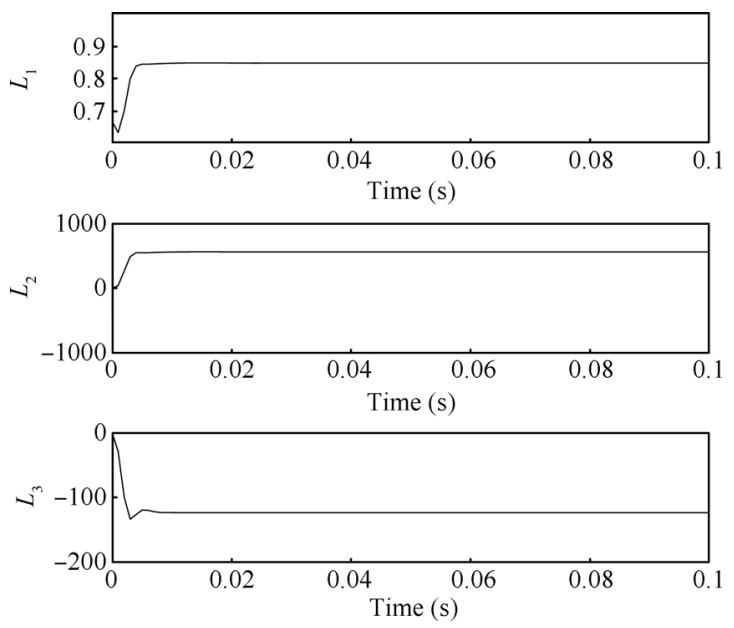

Fig. 5 Convergence of the Kalman gains

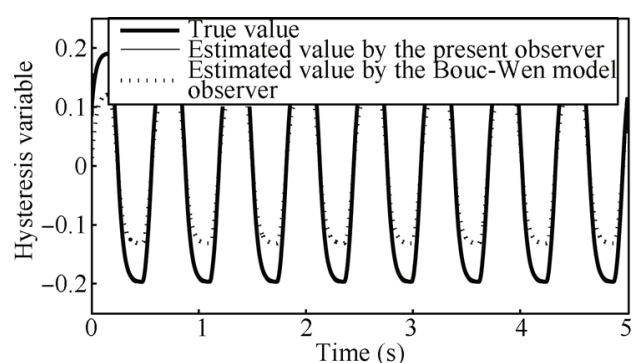

Fig. 6 Simulation results of estimated hysteresis variables with $\alpha$ increased by $50 \%$

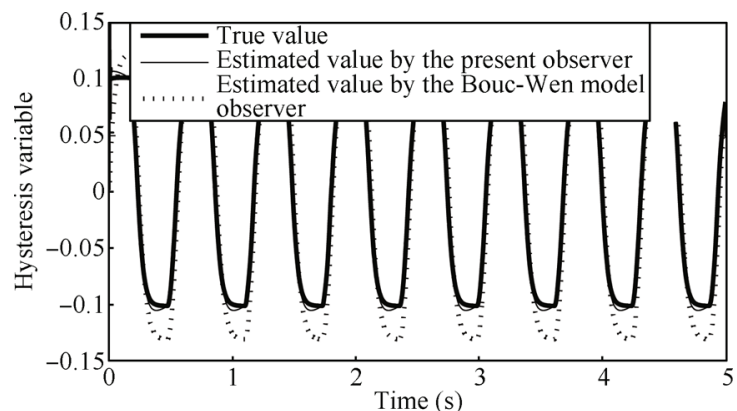

Fig. 7 Simulation results of estimated hysteresis variables with $\beta$ increased by $50 \%$

To demonstrate the potential of the proposed method in control applications, the feedforward compensation method presented in [18] is adopted here. The velocity feedback is also employed for suppression of the high oscillatory behavior of the flexible beam. The control block diagram is summarized in Fig. 9, where $r$ denotes the tracking reference signal and $k_{v}$ is the velocity feedback gain. However, since the PI-observer is closed-loop, the block diagram is slightly modified. The block diagram of the PI-observer based approach is shown in Fig. 10.

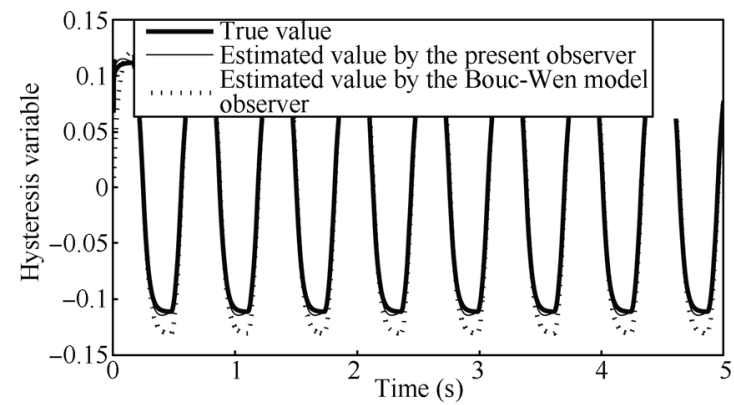

Fig. 8 Simulation results of estimated hysteresis variables with $\gamma$ increased by $50 \%$

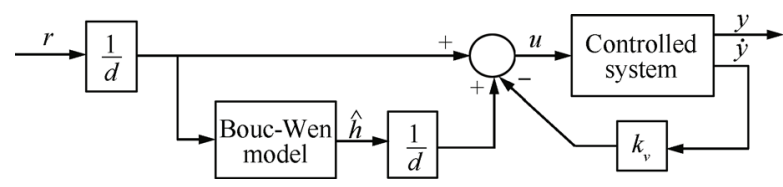

Fig. 9 Bouc-Wen model based feedforward compensating block diagram

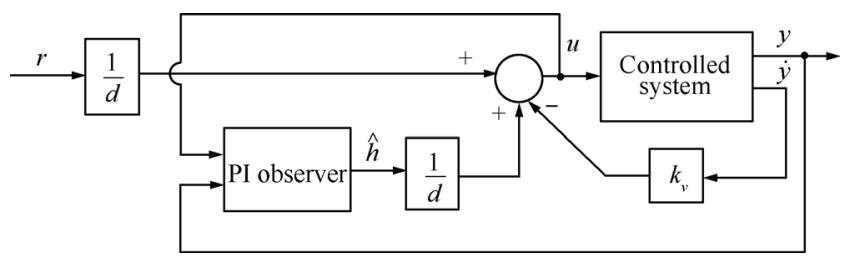

Fig. 10 PI-observer based feedforward compensating block diagram

The simulation results of the feedforward compensation based on the Bouc-Wen model and the proposed method are shown in Figs. 11 and 12. The control objective is to track a triangular reference signal. The gain $k_{v}=0.02$ was chosen. In Fig. 11, it is assumed that the parameters of the 


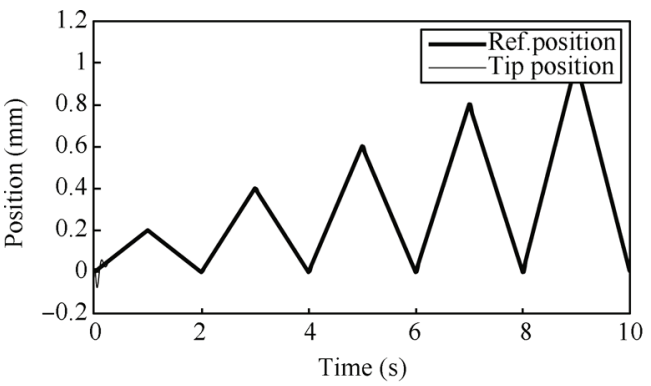

(a) Based on the present method

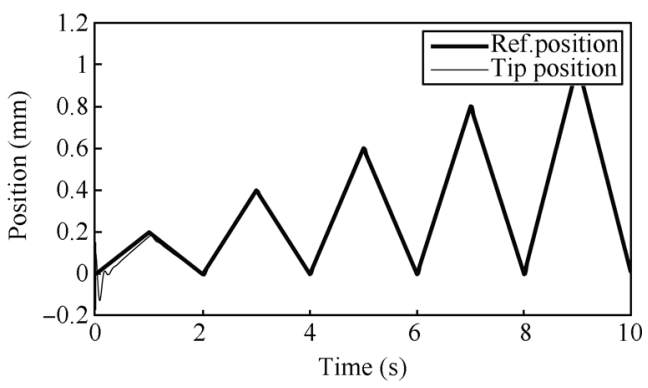

(b) Based on the Bouc-Wen model observer

Fig. 11 Simulation results of feedforward compensating control

Bouc-Wen model are known exactly. In this case, both methods yield similar tracking accuracy. However, when the parameters are not known precisely, the proposed method still gives a satisfactory tracking accuracy whereas the other one results in less accuracy due to the estimation error (see Fig. 12). Note that the effect of estimation error might be reduced by adding a suitable feedback controller as mentioned in [18].

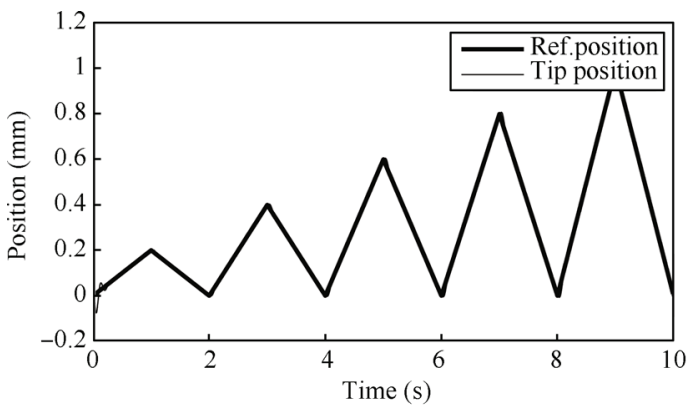

(a) Based on the present method

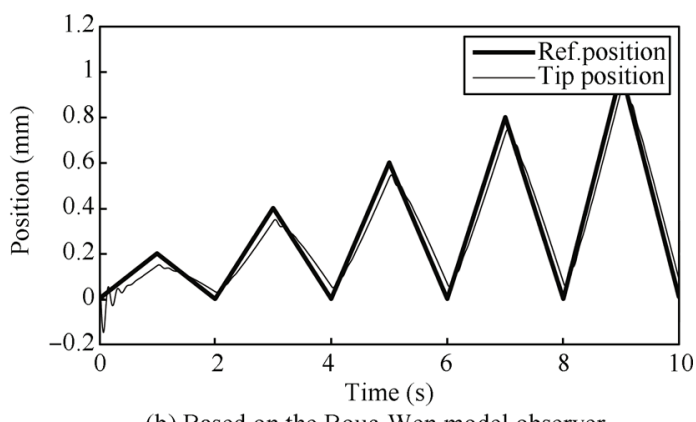

(b) Based on the Bouc-Wen model observer

Fig. 12 Simulation results of feedforward compensating control with $\alpha$ increased by $50 \%$
Remark 1. Similar to the work in [24], the presented method can also be utilized to estimate the state variables of the controlled system for feedback purpose. In Appendix, we show that the present method yields better estimations than the conventional Kalman filter when the hysteresis is included in the system.

\section{$6 \quad$ Experimental results}

The sampling rate of $0.001 \mathrm{~s}$ is used in all experiments. The algorithm is written in C. The same values of all parameters used in the simulation studies are also used in the experiments. As shown in Fig. 13, the present observer estimates the hysteresis loop effectively. The estimated hysteresis variable is shown in Fig. 14. Note that the magnitude of the estimated hysteresis variable is larger than the one found in the simulation studies. This is mainly because the real-life system contains infinite numbers of vibration modes. Thus, the estimation includes both the effects of the hysteresis and the dynamics of all higher vibration modes.

An example of control application using the feedforward compensation is shown in Fig. 15. Here, due to the effect of the higher vibration modes, $\hat{h}$ in the control block diagram was replaced by $\breve{h}=g \hat{h}$, where $g$ denotes the hysteretic effective gain. Based on experimental tunings, $g=0.25$ is chosen. The results show that the tracking error is improved by using the compensation with the present observer. In summary, the present observer is successfully implemented in the real-life system.

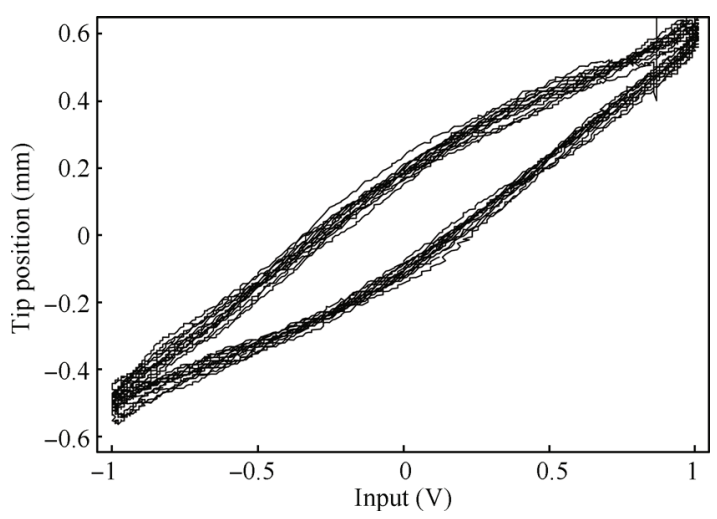

Fig. 13 Estimated hysteresis loop of the experimental system

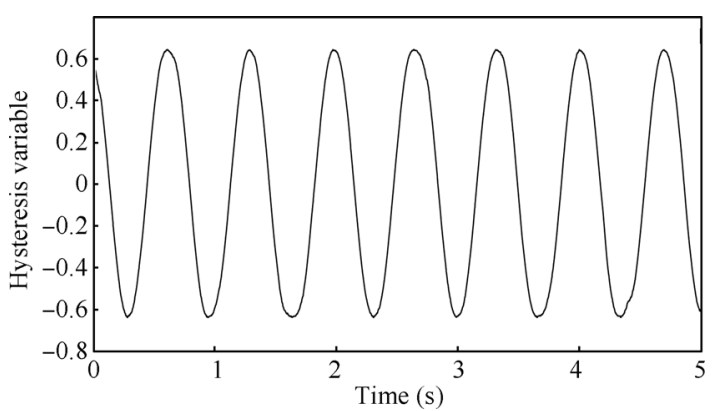

Fig. 14 Estimated hysteresis variable of the experimental system 

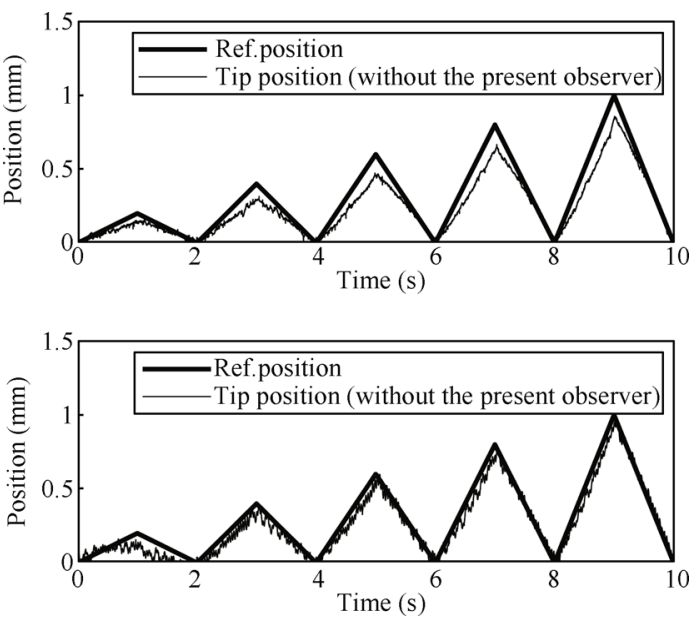

Fig. 15 Experimental results of feedforward compensating control

\section{Conclusions}

This paper proposes a hysteresis observer based on a nonlinearity observer method for piezo-actuated flexible beams. The discrete-time Kalman-filter algorithm is adopted for determination of the observer gains. The simulation studies demonstrate that the observer is able to estimate the hysteresis efficiently and has better robustness compared to the previous scheme existing in the literature. The proposed observer is also successfully implemented on a reallife piezo-actuated aluminum beam. An example of control applications based on feedforward compensation illustrates the effectiveness in both simulation and experimental studies.

\section{Appendix}

In this appendix, a simulation study on utilizing the present method for state estimation is conducted. The model (7) with the parameters in Table 3 is used. The results are shown in Fig. 16, comparing to the ones obtained by using the conventional Kalman filter as done in [24], where the hysteresis is ignored. Here, the input is set as $u(t)=\sin (10 t)$ volts. $Q=\left[\begin{array}{cc}1 & 0 \\ 0 & 1 \times 10^{5}\end{array}\right]$ and $Q=\left[\begin{array}{ccc}1 & 0 & 0 \\ 0 & 1 \times 10^{5} & 0 \\ 0 & 0 & 1 \times 10^{10}\end{array}\right]$ are used for designing the conventional Kalman filter and the present observer, respectively. $R=1$ is chosen in both cases. Note that the estimated state vector for the conventional Kalman filter is $\hat{X}^{\mathrm{T}}=\left[\begin{array}{cc}\hat{y} & \dot{\hat{y}}\end{array}\right]$ whereas the present observer $\hat{X}^{\mathrm{T}}=\left[\begin{array}{ccc}\hat{y} & \dot{\hat{y}} & \hat{h}\end{array}\right]$. Fig. 16 shows that both observers can estimate the tip position very well. However, the present observer significantly outperforms the other for estimation of the tip velocity. This is due to the effect of the hysteresis.
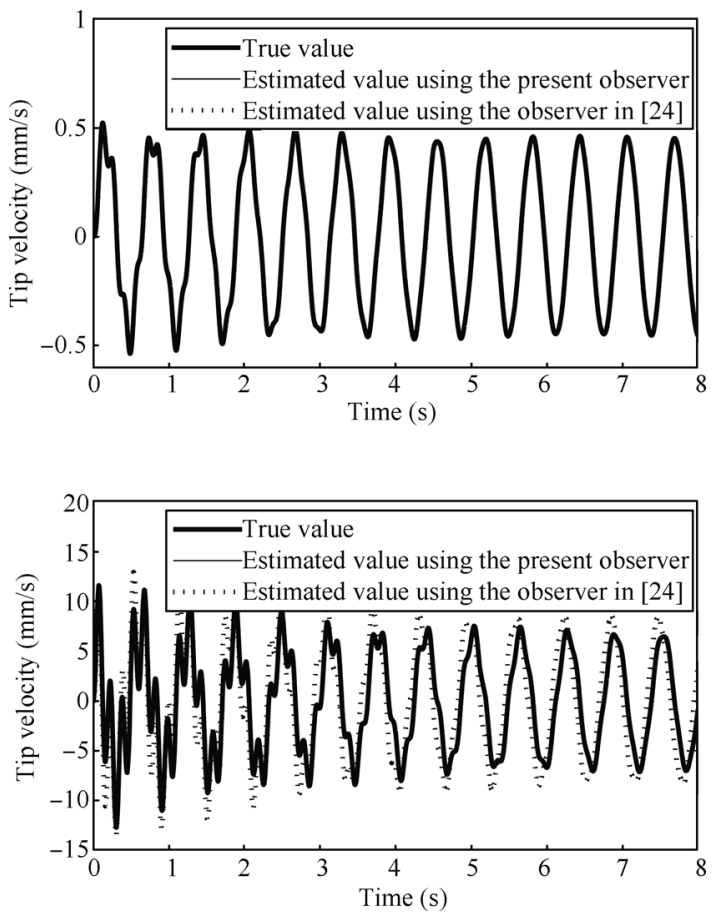

Fig. 16 Simulation results of state estimation

\section{References}

[1] J. F. Cuttiono, A. C. Jr. Miller, D. E. Schinstock. Performance optimization of a fast tool servo for single-point diamond turning machines. IEEE/ASME Transactions on Mechatronics, vol. 4, no. 2, pp. 169-179, 1999.

[2] J. S. Xu, H. Guo. Study on driving and detection of microknife for minimally invasive surgery. In Proceedings of the 5th IEEE International Conference on Nano/Micro Engineered and Molecular Systems, IEEE, Xiamen, China, pp. 919-923, 2010.

[3] M. Kögl, M. L. Bucalem. Analysis of smart laminates using piezoelectric MITC plate and shell elements. Computers and Structures, vol. 83, no. 15-16, pp. 1153-1163, 2005.

[4] H. Bossong, S. Lentzen, R. Schmidt. Experimental investigation and modelling of piezoelectric actuator hystereses for FE analysis of smart structures. Computational Methods and Experimental Measurements XII, C. A. Brebbia, G. M. Carlomagno, Eds., Southampton, UK: WIT Press, pp. 217-226, 2005.

[5] Y. H. Yu, N. Naganathan, R. Dukkipati. Preisach modeling of hysteresis for piezoceramic actuator system. Mechanism and Machine Theory, vol. 37, no. 1, pp. 49-59, 2002.

[6] H. Bossong, R. Schmidt, D. Weichert. Numerical modelling of the hysteretic behaviour of piezoactuated structures. Shell Structures, Theory and Applications, W. Pietraszkiewicz, I. Kreja, Eds., London, UK: Taylor \& Francis, vol. 2, pp. 283-286, 2010.

[7] C. A. Jiang, M. C. Deng, A. Inoue. Robust stability of nonlinear plants with a non-symmetric Prandtl-Ishlinskii hysteresis model. International Journal of Automation and Computing, vol. 7, no. 2, pp. 213-218, 2010.

[8] M. A. Janaideh, P. Krejčí. Prandtl-Ishlinskii hysteresis models for complex time dependent hysteresis nonlinearities. Physica B, vol. 407, no. 9, pp. 1365-1367, 2012.

[9] J. H. Song, A. D. Kiureghian. Generalized Bouc-Wen model for highly asymmetric hysteresis. Journal of Engineering Mechanics, vol. 132, no. 6, pp.610-618, 2006. 
[10] F. Ikhouane, J. E. Hurtado, J. Rodellar. Variation of the hysteresis loop with the Bouc-Wen model parameters. Nonlinear Dynamics, vol. 48, no. 4, pp. 361-380, 2007.

[11] M. Chen, C. S. Jiang, Q. X. Wu. Sensor fault diagnosis for a class of time delay uncertain nonlinear systems using neural network. International Journal of Automation and Computing, vol. 5, no. 4, pp. 401-405, 2008.

[12] K. Mohamed, M. Chadli, M. Chaabane. Unknown inputs observer for a class of nonlinear uncertain systems: An LMI approach. International Journal of Automation and Computing, vol. 9, no. 3, pp. 331-336, 2012.

[13] H. Beikzadeh, H. D. Taghirad. Exponential nonlinear observer based on the differential state-dependent Riccati equation. International Journal of Automation and Computing, vol. 9, no. 4, pp. 358-368, 2012.

[14] L. Freidovich, A. Robertsson, A. Shiriaev, R. Johansson. LuGre-model-based friction compensation. IEEE Transactions on Control Systems Technology, vol. 18, no. 1, pp. 194200, 2010.

[15] L. P. Liu, Z. M. Fu, X. N. Song. Sliding mode control with disturbance observer for a class of nonlinear systems. International Journal of Automation and Computing, vol.9, no. 5, pp. 487-491, 2012.

[16] R. B. Wiener. Nonlinear compensation for pneumatic actuators with hysteresis-precision control for microlithography. IEEE Control Systems Magazine, vol. 25, no.6, pp. 32-44, 2005.

[17] C. Ru, L. Chen, B. Shao, W. Rong, L. Sun. A hysteresis compensation method of piezoelectric actuator: Model, identification and control. Control Engineering Practice, vol. 17, no. 9, pp. 1107-1114, 2009.

[18] C. J. Lin, S. R. Yang. Precise positioning of piezo-actuated stages using hysteresis-observer based control. Mechatronics, vol. 16, no. 1, pp. 417-426, 2006.

[19] L. Juhász, J. Maas, B. Borovac. Parameter identification and hysteresis compensation of embedded piezoelectric stack actuators. Mechatronics, vol. 21, no. 1, pp.329-338, 2011.

[20] L. Liu, K. K. Tan, S. L. Chen, S. Huang, T. H. Lee. SVD-based Preisach hysteresis identification and composite control of piezo actuators. ISA Transactions, vol. 51, no. 3, pp. 430-438, 2012.

[21] P. C. Müller. Estimation and compensation of nonlinearities. In Proceedings of the 1st Asian Control Conference, Tokyo, Japan, vol. II, pp.641-644, 1994.

[22] D. Söffker, T. J. Yu, P. C. Müller. State estimation of dynamical systems with nonlinearities by using proportionalintegral observer. International Journal of Systems Science, vol. 26, no. 9, pp. 1571-1582, 1995.

[23] P. C. Müller. Design of PI-observers and compensators for nonlinear control system. Advances in Mechanics, Dynamics and Control, F. L. Chernousko, G. V. Kostin, V. V. Saurin, Eds., Moscow, Russia: Nauka, pp. 223-231, 2008.

[24] A. Girija, M. Umapathy, B. Bandyopadhyay, G. Uma, K. Dhanalakshmi. Discrete time sliding mode control for piezoelectric actuated structures. In Proceedings of IEEE International Conference on Industrial Technology, IEEE, Mumbai, India, pp. 1466-1471, 2006.
[25] F. Heidtmann, I. Krajcin, D. Söffker. Observer-based control and disturbance compensation of elastic mechanical 2D-/3D-structures. In Proceedings of the 2nd International Conference on Dynamics, Vibration, and Control, IEEE, Beijing, China, pp. 23-26, 2006.

[26] P. Nakkarat, S. Kuntanapreeda. Observer-based backstepping force control of an electrohydraulic actuator. Control Engineering Practice, vol. 17, no. 8, pp. 895-902, 2009.

[27] S. V. Gosavi, A. G. Kelkar. Passivity-based robust control of piezo-actuated flexible beam. Transactions of ASME, vol. 126, no. 2, pp. 260-271, 2004.

[28] A. E. Bryson, Y. C. Ho. Applied Optimal Control: Optimization, Estimation, and Control, New York: Hemisphere, 1975.

[29] R. F. Stengle. Optimal Control and Estimation, New York, USA: Dover, 1994.

[30] G. F. Franklin, J. D. Powell, M. L. Workman. Digital Control of Dynamic Systems, 3rd ed, New York: AddisonWesley, 1997.

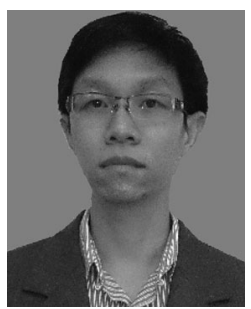

Teerawat Sangpet received the B. Eng. degree in aerospace engineering from King Mongkut's University of Technology North Bangkok (KMUTNB), Thailand in 2008, and M. Eng. degree in mechanical engineering from the same university in 2010 . $\mathrm{He}$ is currently working as a Ph. D. candidate at the Department of Mechanical and Aerospace Engineering, KMUTNB.

His research interests include the areas of nonlinear control and fractional-order control.

E-mail: teerawat.me@gmail.com (Corresponding author)

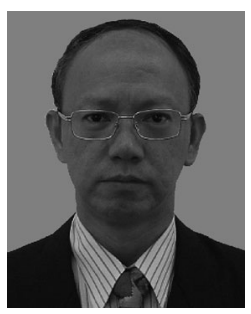

Suwat Kuntanapreeda received the B. Eng. degree in mechanical engineering from King Mongkut's University of Technology North Bangkok (KMUTNB), Thailand in 1990, and the M.S. and Ph. D. degrees in mechanical engineering from Utah State University, USA in 1992 and 1995 , respectively. He is currently an associate professor in the Department of Mechanical and Aerospace Engineering at KMUTNB.

His research interests include the applications of control theory to mechatronic, chaotic, and aerospace systems.

E-mail:suwat@kmutnb.ac.th

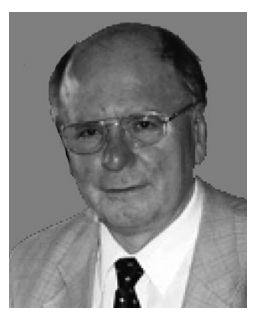

Rüdiger Schmidt received the Diploma of aerospace engineering from RWTH Aachen University, Germany in 1974 and the doctoral degree from the Institute of Mechanics of Ruhr-University Bochum, Germany in 1981 . He is currently a professor at the Institute of General Mechanics of RWTH Aachen University.

His research interests include nonlinear continuum and structural mechanics.

E-mail:schmidt@iam.rwth-aachen.de 